\title{
Synthetic aperture radar raw signal simulator for both pulsed and FM-CW modes
}

\author{
P. Serafin, C. Lesnik \& A. Kawalec \\ Institute of Radioelectronics, Military University of Technology, Poland
}

\begin{abstract}
Simulated raw radar signals prove to be very useful at the first stages of testing radar signal processing algorithms and procedures. This is particularly true for synthetic aperture radar (SAR), where the costs of real signals acquisition are very high due to the costs of building the system as well as the costs of mission (air or space-borne). This paper describes a multifunctional SAR raw signal simulator that has been used for the verification of SAR image synthesis algorithms. Generated signals can be imitated for pulsed as well as FM-CW radars both in SLAR and squinted cases, it is also possible to choose between video and intermediate frequency signals. The simulator allows us to generate echo signals from stationary and moving targets. The user is able to differentiate the statistic properties of received echo signals for each target, thus allowing us to generate different types of reflecting surfaces. If present, a real raw SAR signal can be merged with a simulated one to produce more complicated scenarios. The paper presents results of the simulation of raw signals and their image after SAR processing.
\end{abstract}

Keywords: synthetic aperture radar, SAR signal simulator, FM-CW SAR.

\section{Introduction}

Airborne and space borne radar imaging allows us to collect the pictures of a terrain fragment independently of the time of the day, the weather or visibility conditions over the imaged scene. Unfortunately, due to relatively long waves used by the radar (compared to photography), it needs to employ large antennae in order to achieve the desired image resolution. This can make the system impossible to build onboard an airplane or a satellite. The solution to this problem is the technique of the synthetic aperture radar (SAR) that is able to 
generate "virtual" antenna by scanning the observed area along a known route. After covering a desired distance gathered signals are processed as though they came from a real antenna giving a radar image with the resolution comparable with the wavelength of used signals.

Due to its advantages, SAR systems became very useful and widely applied reconnaissance tools and thanks to the rapid development of the computational techniques, their capabilities have grown steadily over past years.

The development of SAR signal processing algorithms requires the verification of their results with test signals. This can be difficult in the early stages of the project, especially during system feasibility verification, when no physical realisation of the radar that would provide data exists. Acquiring the signals from third party institutions if possible is rather expensive due to the expenses put into building the systems as well as conducting the mission.

Therefore one of the basic sources of radar signals used in SAR image synthesis algorithms testing is the computer simulations. Simulated signals are incomparably cheaper than real ones and their additional advantage is the ability to configure the observed scene and the radar mission arbitrarily, which allows us to test the algorithms in a wide range of parameters.

The main drawback of simulations, however, is the simplifications made in order to keep computational costs as low as possible, which sometimes render the results unreliable. The simulation design must be then optimised for maximum reality and minimum complexity.

This paper presents a simulated SAR raw signal generator developed for the verification SAR image synthesis algorithms in Military University of Technology (MUT) in Warsaw, Poland.

\section{Principle of SAR}

A SAR system is typically a radar installed onboard an airplane or a spaceship whose antenna system is illuminating the Earth's surface perpendicularly or at some squint angle to the carrier's route.

A typical SAR configuration for the airborne case is Side-looking Airborne Radar (SLAR) whose geometry is presented in fig. 1.

The carrier is moving along a set, well known and, preferably, straight, route. During the operation, as its carrier moves the radar emits the electromagnetic energy and receives echo signals reflected from the terrain objects. Received signals are then pre-processed and stored in system memory.

This signal pre-processing includes filtration and down-conversion to intermediate frequency or can be extended to baseband conversion and subsequently range compression, depending on the type of the algorithm used.

After the radar has covered the distance equal the antenna length $L$ needed to achieve the desired azimuth resolution $\rho_{A}$, signals stored in memory are processed as if they were received by a real phased array of a length $L$. 

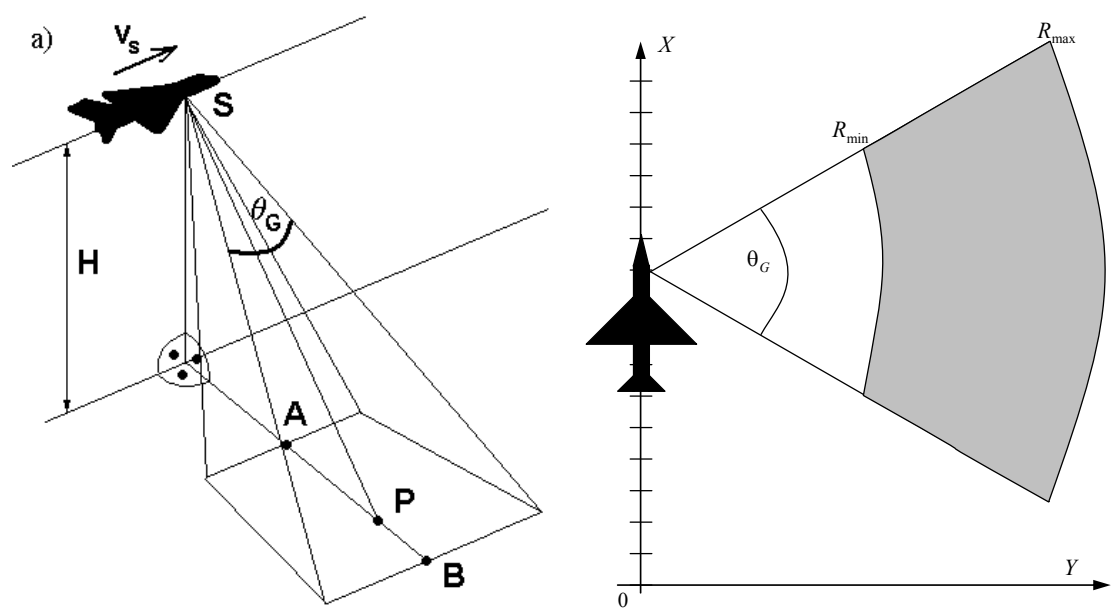

Figure 1: The geometry of a SAR system.

Let us assume the transmitted sounding signal to be of form

$$
s_{T}(t)=A(t) e^{-j\left(\omega_{0} t\right)},
$$

where: $A(t)$ is a modulation signal, $\omega_{0}$ is the centre angular frequency.

Then the echo signal reflected from a single point target at the range $R$ will take form [1]

$$
s(t)=s_{T}(t-\tau) e^{-j \frac{4 \pi}{\lambda} R},
$$

where: $\lambda$ is the wavelength of sounding signal, $\tau$ is a two way electromagnetic wave propagation time between the radar antenna and the target given by

$$
\tau=\frac{2 R}{c}
$$

where $c$ is the wave propagation velocity.

If one takes into account that the radar is moving and denote the time $t$ associated with wave propagation as "fast-time" and the time $T$ associated with the radar movement, as "slow-time" one will obtain the complete equation describing received signal

$$
s(T, t)=s_{T}(t-\tau(T)) e^{-j \frac{4 \pi}{\lambda} R(T)} .
$$


In the above equation the instantaneous (in the slow-time) range $R(T)$ is defined as follows

$$
R(T)=\sqrt{\left(x_{R}(T)-x_{T}\right)^{2}+\left(y_{R}(T)-y_{T}\right)^{2}+\left(z_{R}(T)-z_{T}\right)^{2}},
$$

where: $\left(x_{R}(T), y_{R}(T), z_{R}(T)\right)$ is the instantaneous radar position and $\left(x_{T}, y_{T}, z_{T}\right)$ is the position of the target.

Assuming the coordination system from fig. 1 one can define the coordinates as:

$$
\begin{gathered}
x_{R}(T)=x_{0}+v_{R} T, \\
y_{R}(T)=\text { const }=0, \\
z_{R}(T)=\text { const }=H_{R},
\end{gathered}
$$

where: $x_{0}$ is the initial radar position along the $X$ axis, $v_{R}$ is the radar speed and $H_{R}$ is the radar height above the ground. It is also assumed that the target lies on the ground meaning

$$
z_{T}=0 .
$$

Therefore one can reduce eqn (5) to

$$
R(T)=\sqrt{\left(x_{0}+v_{R} T-x_{T}\right)^{2}+y_{T}^{2}+H_{R}^{2}}
$$

and then substitute the result to eqn (4) which takes then the following form

$$
s(T, t)=s_{T}\left(t-\frac{2 R(T)}{c}\right) e^{-j \frac{4 \pi}{\lambda} \sqrt{\left(x_{0}+v_{R} T-x_{T}\right)^{2}+y_{T}^{2}+H_{R}^{2}}} .
$$

If the signal is sampled with sampling frequency $f_{s}$ and define the range resolution cell dimension as

$$
d R=\frac{c}{2 f_{s}},
$$

one can obtain

$$
s(m, l)=s_{T}\left(\left\lfloor l-\frac{R(T)}{d R}\right\rfloor\right) e^{-j \frac{4 \pi}{\lambda} \sqrt{\left(x_{0}+l d-x_{T}\right)^{2}+y_{T}^{2}+H_{R}^{2}}},
$$


where: $m$ is a number of the sounding impulse emitted by the radar with the Pulse Repetition Frequency $(P R F), l$ is a number of range cell and $d$ is distance between two consecutive sounding positions of the radar

$$
d=\frac{v_{R}}{P R F} .
$$

In order to obtain a high resolution radar image one should compress the signal in the range (fast-time) and azimuth (slow-time) domains.

The range compression is performed by a filter matched to the form of the sounding signal $s_{T}$ and can be done either before the azimuth compression or after it. Some of the SAR image synthesis algorithms combine those two compressions in one block. They will be, however, considered here as separate processing steps.

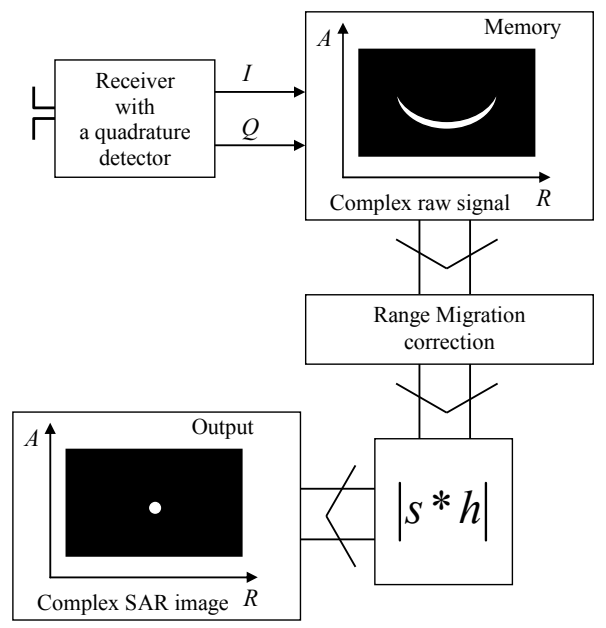

Figure 2: $\quad$ Structure of the TDC SAR processor.

It will be assumed that the obtained raw SAR signal is already rangecompressed.

The azimuth compression is in fact matched filtering [1, 2], where filter impulse response is so called "azimuth chirp" being actually the complex conjugate to the exponential form in eqn (13).

Therefore the operation of azimuth compression of the SAR image would be a convolution of the signal with the azimuth chirp. However simultaneously with the changes of signal phase due to the changes of range the signal position in system memory changes as well. This phenomenon, called Range Migration (RM), if not compensated for seriously decreases maximum achievable synthetic aperture length and consequently the maximum image resolution. Range Migration correction procedure consists of computing the actual number of range 
cell where the signal for a given image pixel for each element of the synthetic aperture.

Therefore the structure of the SAR processor (shown in fig. 2) consists the above mentioned convolution block following the RM correction step.

The described algorithm is called Time Domain Correlation (TDC) and although it is computationally very expensive, it is also the most accurate due to the lack of any simplifying assumptions. This makes this algorithm a useful reference tool for verification of other SAR algorithms.

\section{The simulation strategy}

The simulated raw SAR signal generator developed in MUT was designed to evaluate the accuracy and efficiency of SAR algorithms implemented in an FPGA structure.

The generator can provide the single- or multi-channel raw SAR signals reflected from user-defined objects placed in the observed space. Objects are flat and lay on the Earth surface. They can, however, move in the $X$ and $Y$ directions.

Each object consists of a number of elementary scatterers distributed within its boundaries.

User can define the shape (rectangle, ellipse or line) of the object, its size, the number of scatterers within the object and the $v_{x}$ and $v_{y}$ velocity components.

There are three types of objects in the program:

1) with randomly distributed scatterers that have defined parameters such as minimal and maximal values of the Radar Cross Section (RCS) over the object, variance of RCS and phase fluctuations of all scatterers from pulse to pulse; additionally each scatterer can have omnidirectional scattering characteristic in the azimuth and elevation domain or it can have an eight shaped one in azimuth domain; in the latter case user can also define the range of angles for azimuthal position of the scatterers; the probability density function (PDF) for RCS fluctuations is assumed to be Gaussian, whereas the phase distribution is uniform and those fluctuations are uncorrelated,

2) with uniformly distributed elementary reflecting surfaces; the statistical properties of such object are described by PDF and autocorrelation function (AF) of those surfaces,

3) jamming sources emitting white Gaussian noise with an omnidirectional characteristic.

The user has also to define all the radar parameters such as number of receiving channels $q_{\max }$, distance between the antenna elements $d_{a}$, centre frequency $f_{0}$ and wavelength $\lambda$, pulse repetition frequency $P R F$, LFM deviation $\Delta f$, pulse duration $T_{i}$, sampling frequency $f_{s}$, intermediate frequency $f_{I M}$ and the emitted peak power. Also the carrier parameters such as 
height, velocity, observation distance or initial coordinates need to be established.

Radar can have a number of receiving channels whose antennae are uniformly distributed along the carrier movement direction.

The sounding signal in the simulated SAR system can be either pulsed or continuous wave with linear frequency modulation (LFM-CW), the raw signal after simulation will differ in each case. For the pulsed radar it is either video or IM signal with or without range compression, and for the LFM-CW case it is the Discrete Fourier Transform (DFT) of dechirped (down-converted) received signal. Both types of signal are stored in a text or a binary file as complex samples arranged in range lines (signals received during one sounding).

The user can also define the standard deviation of the receiver thermal noise, which will have added to the signal at the end of the simulation.

\section{The simulation algorithm}

The echo signal reflected from each element is computed accordingly to the following algorithm

1) For each $m$-th position of radar (for each sounding) the distance $R_{m, n}$ between the radar antenna and the $n$-th scatterer is computed accordingly to the following equation

$$
R_{m, n}=\sqrt{\left(x_{R m}-x_{T m, n}\right)^{2}+\left(y_{R m}-y_{T m, n}\right)^{2}+\left(H_{R}-h_{T m, n}\right)^{2}},
$$

where: $x_{R m}$ and $y_{R m}$ are the radar position coordinates in the $m$-th sounding, and $x_{T m, n}$ and $y_{T m, n}$ are the coordinates of the $n$-th scatterer in the $m$-th sounding, $H_{R}$ is the height of the carrier above the ground.

If the position of no. 0 antenna element would be assumed as the position of radar $x_{R m}$, then the position of the $q$-th antenna element can be defined as

$$
x_{a q m}=x_{R m}-q d_{a} .
$$

As it was mentioned all the objects are flat and are placed at the height equal

$$
h_{T m}=h_{T}=0 \text {. }
$$

Considering the above and the radar movement the statement for the distance between $n$-th scatterer and $q$-th antenna element in the $m$-th sounding can be rewritten as follows

$$
R_{m, n, q}=\sqrt{\left(x_{R 0}-q d_{a}+m d-x_{T m, n}\right)^{2}+\left(y_{R m}-y_{T m, n}\right)^{2}+H_{R}^{2}} .
$$


As in accepted model the radar $Y$ coordinate $y_{R m}$ is constant and equal to zero, and the objects can be moving with the velocity having components $v_{T x}$ and $v_{T y}$ the eqn (18) should take the following form

$$
R_{m, n, q}=\sqrt{\left(x_{R 0}-q d_{a}+m d-\left(x_{T n 0}+\frac{m v_{T n x}}{P R F}\right)\right)^{2}+\left(\frac{m v_{T n y}}{P R F}-y_{T n 0}\right)^{2}+H_{R}^{2}},
$$

where: $x_{T n 0}$ and $y_{T n 0}$ are the coordinates of the $n$-th scatterer.

2) The received echo signals reflected from the simulated objects are created either as impulses with linear frequency modulation (LFM) or the LFM-CW signals after dechirping. The pulsed signals can have zero central frequency (video signals) or else their spectrum can be centred around a non-zero intermediate frequency $f_{I M}$. The video signals are assumed to be after quadrature down-conversion and therefore are stored as complex samples whereas signals with nonzero $f_{I M}$ are real with imaginary part equal to zero.

3) After having computed the distance $R_{m, n, q}$, voltage samples in receiving channel are determined

$$
U_{m, n, q}\left(l t_{s}\right)=A_{m, n, q} s_{T}\left(l t_{s}-t_{m, n, q}\right) e^{-j \frac{4 \pi R_{m, n, q}}{\lambda}},
$$

where: $s_{T}(t)$ is the function describing the transmitted sounding signal, $t_{m, n, q}$ is the time of the two way propagation of the electromagnetic wave from the $q$-th antenna element to the $n$-th scatterer and back to antenna in the $m$-th sounding and is equal

$$
t_{m, n, q}=\frac{2 R_{m, n, q}}{c}
$$

$A_{m, n, q}$ is the amplitude of the echo signal dependant on scatterer reflecting characteristic $\sigma_{m, n, q}$ (RCS), antenna characteristic $G_{A}$, range and the transmitted power $P_{N}$ and is equal to [2]

$$
A_{m, n, q}=\frac{G_{A}^{2} \lambda}{R_{m, n, q}^{2}} \sqrt{\frac{P_{N} \sigma_{m, n, q}}{(4 \pi)^{3}}} .
$$


The form of the transmitted sounding signal $s_{T}(t)$ is dependant on radar parameters set by the user. In particular if the value of $f_{I M}$ is non-zero this signal is generated as a series of real valued samples having the form of

$$
s_{T}(l)=\cos \left(2 \pi\left(f_{I M}-\frac{\Delta f}{2}+\frac{\Delta f}{2 T_{i}} l t_{s}\right) l t_{s}\right) .
$$

In the case of the baseband (i.e. video) signals it takes the form of a series of complex valued samples

$$
s_{T}(l)=\cos \left(2 \pi\left(\frac{\Delta f}{2 T_{i}} l t_{s}-\frac{\Delta f}{2}\right) l t_{s}\right)+j \sin \left(2 \pi\left(\frac{\Delta f}{2 T_{i}} l t_{s}-\frac{\Delta f}{2}\right) l t_{s}\right) .
$$

If the scatterer under consideration is not fluctuating and it has an omnidirectional reflecting characteristic the $\sigma_{m, n, q}$ parameter is constant. However for scatterers with eight-shaped reflecting characteristic a $\theta_{m, n, q}$ angle between radar-target line and the azimuthal position of the scatterers characteristic is established and then on its basis the $\sigma_{m, n}\left(\theta_{m, n}\right)$ is computed.

The signal can be range compressed by matched filtering in a FIR filter whose coefficients are the samples of the sounding pulse replica. The filtering itself if done in the form of so called fast convolution employing the Fast Fourier Transform (FFT) on both signal and the impulse replica, then multiplication of the spectra, and finally the Inverse Fast Fourier Transform (IFFT).

In the case of LFM-CW radar the received signal is computed as the result of downconversion with the transmitted signal and for a single scatterer it takes the form of real valued series of samples [3]

$$
U_{m, n, q}\left(l t_{s}\right)=A_{m, n, q} \cos \left(2 \pi\left(f_{0} \tau+\mu\right) \tau l t_{s}-\frac{\mu\left(l t_{s}\right)^{2}}{2}\right) .
$$

After computing the values of the signal samples and their position in the system memory the signals are added to the respective cells (their values are summed with those already existing it the memory).

If simulated radar is working as LFM-CW the FFT in the range domain is performed in order to obtain the range compression.

The program has the functionality of synthesising the SAR image using described earlier the TDC algorithm, which can be used as the tool for verification of the generated raw signals. It is also possible to merge new situation with earlier generated signals or even with real radar signals if only all of the needed parameters are known. 


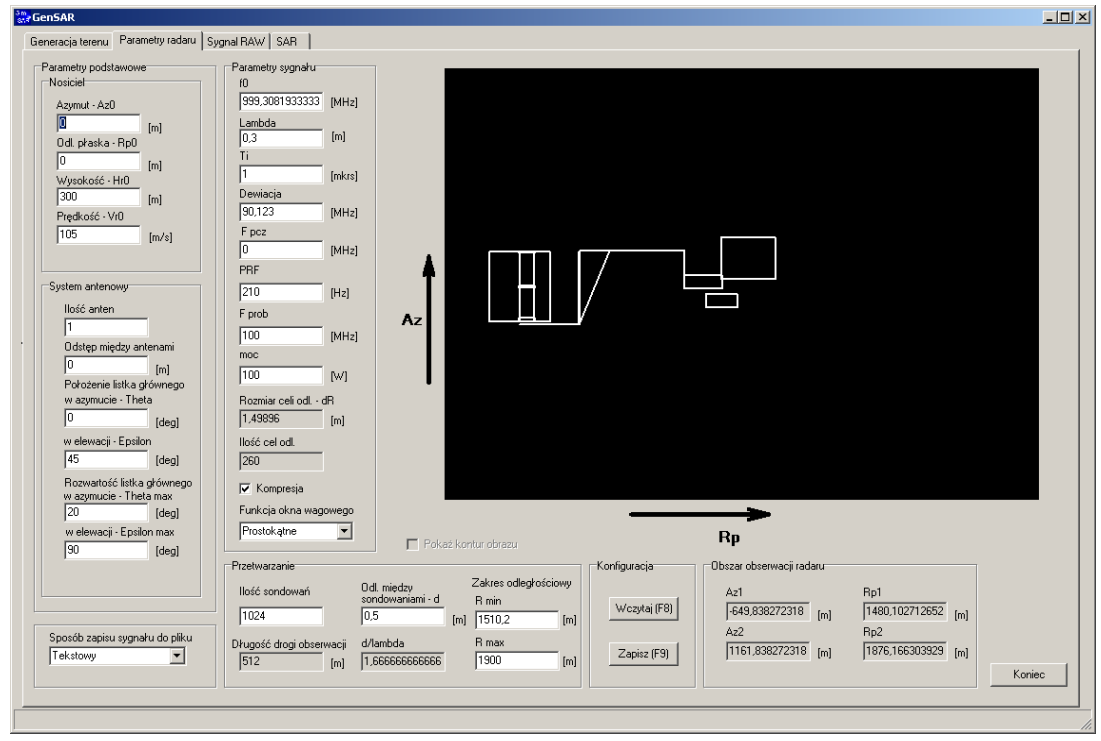

Figure 3: $\quad$ Program window with radar parameters for the simulation tab.

Fig. 3 presents the program window with radars parameters tab showing also the distribution of the simulated objects. In fig. 4 the tab with generated raw SAR signal is presented and in fig. 5 the tab with SAR image computed with the TDC SAR image synthesis algorithm.

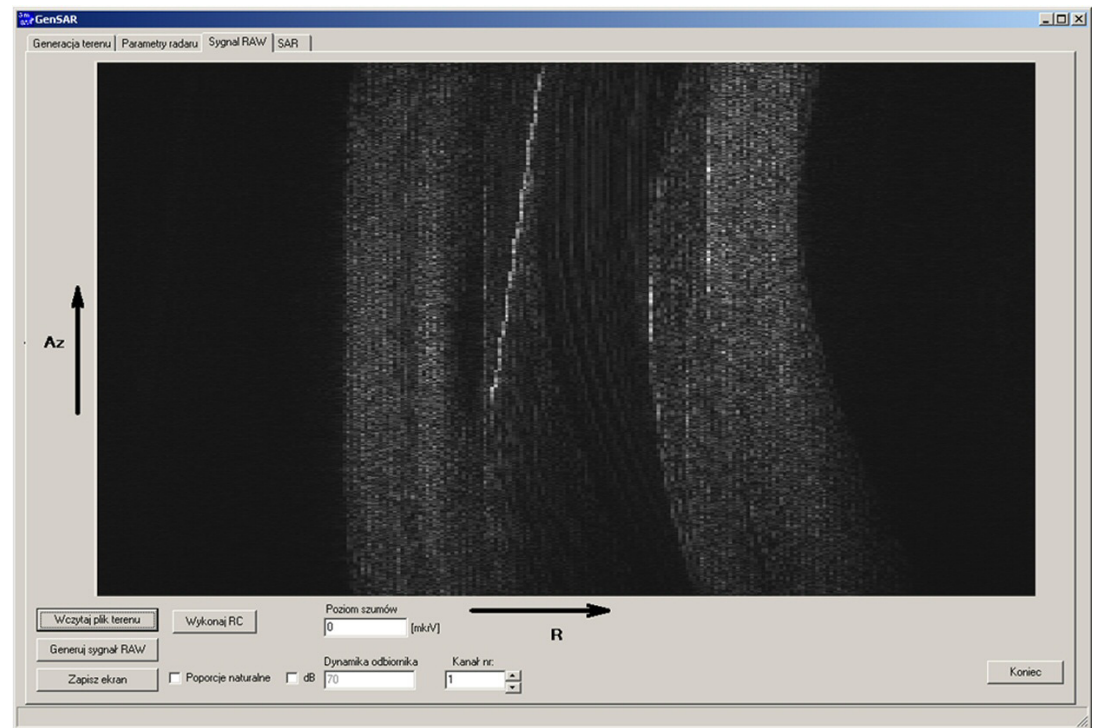

Figure 4: $\quad$ Program window with SAR raw signal generated. 


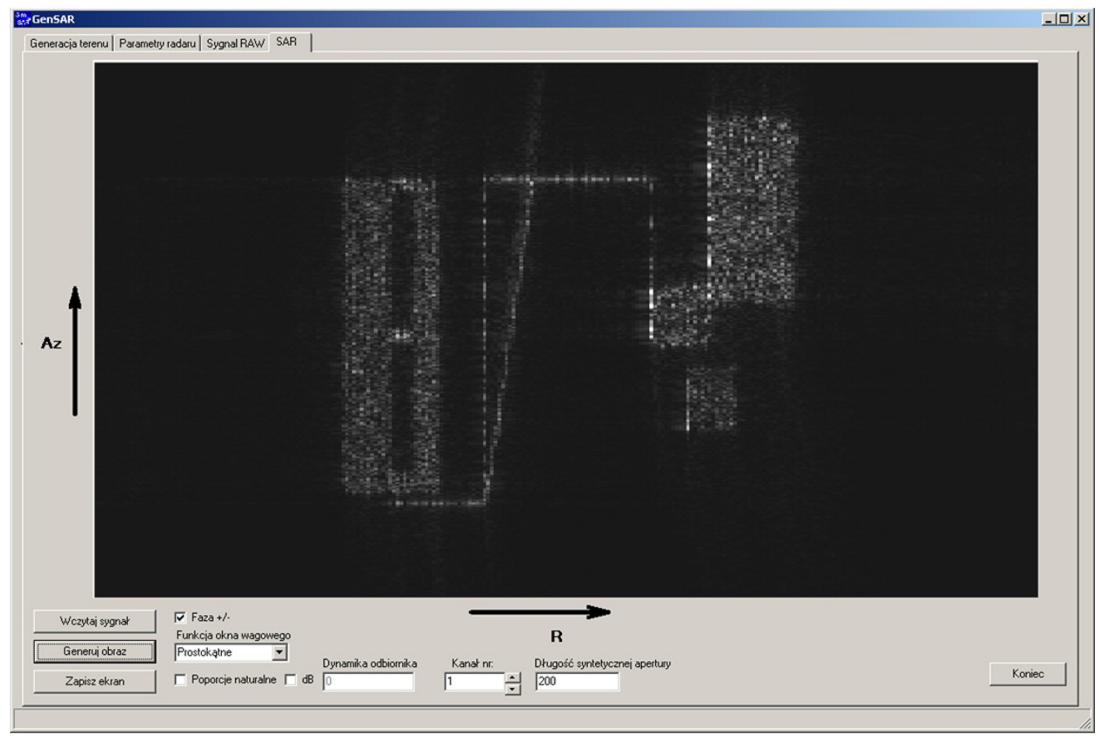

Figure 5: $\quad$ Program window with SAR image synthesised.

\section{Conclusions}

A Synthetic Aperture Radar raw signal generator developed in Military University of Technology in Warsaw, Poland was descried in this paper. Algorithm of the generation of echo signals from both stationary and moving targets was presented as were the simulation results.

\section{Acknowledgement}

This work was supported by the Polish Ministry of Science and Higher Education from sources for science in the years 2009-2011 under project OR00007509.

\section{References}

[1] Cumming, I.G. \& Wong, F.H., Digital Processing of Synthetic Aperture Radar Data. Algorithms and Implementation, Artech House: London and Boston, 2005.

[2] Skolnik, M., Radar Handbook, Second Edition, McGraw-Hill Book Company: New York, 1990.

[3] Stove, A.G., Linear FMCW radar techniques. IEE Proceedings $F$ Radar and Signal Processing, 139(5), pp. 343-350, 1992. 